\title{
Joint Scheduling for Dual-Hop Block-Fading Broadcast Channels
}

\author{
Ammar Zafar*, Mohammad Shaqfeh ${ }^{\star}$, Mohamed-Slim Alouini*, and Hussein Alnuweiri \\ *EE Program, King Abdullah University of Science and Technology (KAUST), Thuwal, Makkah Province, Saudi Arabia \\ Emails:\{ammar.zafar, slim.alouini\}@kaust.edu.sa \\ ${ }^{\star}$ Department of Electrical and Computer Engineering, Texas A\&M University at Qatar, Doha, Qatar \\ Emails:\{mohammad.shaqfeh, hussein.alnuweiri\}@qatar.tamu.edu
}

\begin{abstract}
In this paper, we propose joint user-and-hop scheduling over dual-hop block-fading broadcast channels in order to exploit multi-user diversity gains and multi-hop diversity gains all together. To achieve this objective, the first and second hops are scheduled opportunistically based on the channel state information and as a prerequisite we assume that the relay, which is half-duplex and operates using decode-and-forward, is capable of storing the received packets from the source until the channel condition of the destined user becomes good to be scheduled. We formulate the joint scheduling problem as maximizing the weighted sum of the long term achievable rates by the users under a stability constraint, which means that on the long term the rate received by the relay should equal the rate transmitted by it, in addition to constant or variable power constraints. We show that this problem is equivalent to a single-hop broadcast channel by treating the source as a virtual user with an optimal priority weight that maintains the stability constraint. We show how to obtain the source weight either off-line based on channel statistics or on real-time based on channel measurements. Furthermore, we consider special cases including the maximum sum rate scheduler and the proportional fair scheduler. We demonstrate via numerical results that our proposed joint scheduling scheme enlarges the rate region as compared with a scheme that employs multi-user scheduling alone.
\end{abstract}

Index Terms-Broadcast channel, dual-hop, multi-hop diversity, multi-user diversity, joint scheduling.

\section{INTRODUCTION}

In the Long Term Evolution (LTE)-Advanced systems, fixed access point relays with only an in-band wireless connection to the backhaul network are to be deployed in order to enhance the system capacity and coverage [1]. One of the major challenges for LTE-Advanced systems is to utilize the precious air-link (i.e. bandwidth) resources efficiently to enable achieving the prospected high Quality-of-Service (QoS) requirements [2]. Therefore, the resource allocation task is of crucial importance and we aim to investigate this problem taking into consideration the use of the relays in LTEAdvanced systems.

Two relay functionalities are considered in LTE-Advanced [1]; (i) Transparent relays in which a user has a direct link

This paper was made possible by YSREP grant \# 2-011-2-002 from the Qatar National Research Fund (a member of Qatar Foundation). Furthermore, KAUST funded the efforts of A. Zafar partially and the efforts of M.S. Alouini. The statements made herein are solely the responsibility of the authors. with the base station in addition to the relay link, and (ii) Nontransparent relays which extend the coverage of a cell to reach remote users with no connection to the base station. The latter follows a dual-hop channel model. In [3], the optimal joint power and resource allocation for the transparent relays case was considered. The problem was formulated by maximizing the achievable rate region of block-fading relay-assisted broadcast channels (i.e. for the downlink). The relays were assumed to operate using decode-and-forward (DF). Closedform formulas were provided to decide when to seek relay support and how to integrate this with the opportunistic multiuser scheduling task. In [4], the uplink case was discussed and optimal resource allocation to maximize the achievable rate region of relay-assisted multiple access channels was obtained. However, constant channel model was applied.

In this paper, we consider the downlink of a multi-user network with the users connected to the source through a non-transparent relay under block-fading channels. Hence, there are no direct connections between the source and the users. Our contribution is providing the optimal solution to exploit multi-user diversity (MUD) gains and multihop diversity (MHD) gains jointly. We propose a novel joint userand-hop scheduling scheme that opportunistically allocates the resources based on instantaneous channel measurements. We provide rigorous discussion about the proposed scheduler. In particular we show how to optimize it off-line based on channel statistics or on real-time based on channel measurements. Moreover, we provide closed-form formulas to characterize the long-term average achievable rates via the proposed scheduler. Also, we discuss the scheduling metric in case of constant versus variable power allocation. Furthermore, we apply the joint scheduling scheme for the common maximum sum throughput scheduler and the proportional fair scheduler. On top of that, in order to characterize the obtained MHD gains, we compare the performance versus a benchmark with no MHD gains. Specifically, we compare the proposed joint scheduler with a conventional scheme that applies multi-user scheduling alone yielding a MUD gain and a buffering (at the relay) gain but not full multi-hop diversity gain. We provide thorough numerical results to demonstrate our results and findings.

The rest of the paper is organized as follows. Section II describes the system model and the problem formulation. In Section III, we discuss a scheduling scheme with user schedul- 


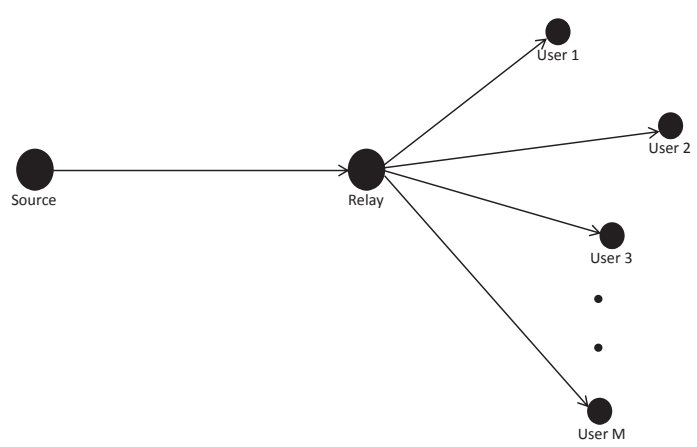

Fig. 1: System model: dual-hop broadcast channel.

ing alone. Next, we discuss the proposed joint scheduling scheme in Section IV. The numerical results are presented in Section V. Finally, Section VI concludes the paper.

\section{SySTEM MODEL}

Consider a system with a source $(\mathrm{S})$, a relay $(\mathrm{R})$ and $M$ users or destinations (D). There are no direct links between the source and the $M$ users. Hence, the source transmits to the users only through the relay as shown in Fig. 1. A block-fading channel model is assumed. One channel block, called a resource unit (RU), can consist of multiple time slots and multiple frequency sub-carriers. However, the RUs are orthogonal to each other (i.e. they do not overlap over time or frequency domain). The relay is assumed to work in halfduplex mode, which means that the relay cannot receive and transmit simultaneously over the same RU. Furthermore, the relay follows the decode-and-forward (DF) protocol. The fading gains of the S-R link and the R-D links stay constant over a single RU. However, they change randomly and independently from one RU to another. Moreover, the channel gains of all the links, i.e. S-R and R-D, experience independent fading. Without loss of generality we assume that all RUs have the same duration and bandwidth. We also assume that a single user only can be allocated in one RU.

The relay has channel state information (CSI) of its channels with all users and with the source. The objective is to exploit the channel knowledge in the resource allocation task in order to maximize the achievable rates by obtaining multiuser diversity (MUD) and multi-hop diversity (MHD) gains. To achieve this target, we propose to apply joint user-andhop scheduling scheme in which the first and second hops are scheduled opportunistically based on CSI. As a prerequisite, we assume that the relay has the ability to store the received messages from the source in buffers until the channel of the destined user becomes good and this user gets scheduled. We refer to this as a buffering (Buff) gain, which is an elementary component to obtain full (MHD) gains via joint scheduling (JS). Furthermore, we assume that when the source is scheduled, it may combine the packets of more than one user together within one RU, and the relay distributes the source message across the data buffers for each user. The source and the relay adjust the transmission rates based on the instantaneous channel condition (capacity). Moreover, we assume best effort data traffic so that there are no delay constraints and the objective function is to maximize the longterm average achievable rate (throughput).

In the numerical results provided in Section $\mathrm{V}$, we assume that all nodes are equipped with a single antenna. However, extending the results into the multiple-antenna case is straightforward. The achievable rate (in bits/sec/Hz) over one RU is

$$
R_{i}[k]=\log _{2}\left(1+\frac{P[k]\left|h_{i}[k]\right|^{2}}{N_{0}}\right),
$$

where $h_{i}[k]$ is the channel gain between the relay and the $i$ th user in the $k$ th RU, $P[k]$ is the power in $(\mathrm{Joul} / \mathrm{sec} / \mathrm{Hz})$ and $N_{0}$ is the noise power spectral density. For simplicity of notation, we use a single index $k$ to denote the RUs although they span the time and frequency domains.

We formulate the resource allocation optimization problem as maximizing the weighted sum average ${ }^{1}$ throughput of the $M$ users. We need to apply a stability constraint meaning that the sum throughput to the users cannot exceed the throughput generated by the source. Moreover, we consider two cases in terms of power allocation. For constant power allocation we have $P[k]=\bar{P}$, while for optimal power allocation we have a maximum average (over all RUs) sum (for source and relay) power constraint $\bar{P}$. The main optimization problem (with both stability and power constraints) can be written as

$$
\begin{aligned}
& \max \sum_{i=1}^{M} \mu_{i} \bar{R}_{i}, \quad \text { subject to } \\
& \bar{R}_{0}=\sum_{i=1}^{M} \bar{R}_{i}, \quad \text { and } \quad \frac{1}{K} \sum_{k=1}^{K} P[k] \leq \bar{P},
\end{aligned}
$$

where we give the S-R channel the index zero. $\bar{R}_{0}$ and $\bar{R}_{i}$ are the average throughput of the source and $i$ th user respectively. We will provide the solution for generic users' weights $\mu$, and we will emphasize two special cases $^{2}$ which are maximum sum throughput (all $\mu$ 's are equal) and proportional fairness $\left(\mu_{i}=1 / \bar{R}_{i}\right)[6]$. For now, we will neglect the power constraint and use $P[k]=\bar{P}$. The power constraint will be considered in Section IV-B.

As a benchmark, we consider a scheme which reserves part of the total bandwidth to the source and the remaining part to the relay, and the relay applies opportunistic scheduling over the users' channels. Fig. 2 clarifies the difference between joint hop-and-user scheduling and multiuser-only scheduling. The figure shows an example of how the RUs can be allocated for a system with three users, i.e. $M=3$.

\section{Multi-User Scheduling ONLY}

\section{A. Conventional Scheme with given Weights}

In this scheme (refer to Fig. 2(b)), we can obtain MUD gains due to multiuser scheduling and a buffering gain which

\footnotetext{
${ }^{1}$ We use average and long term interchangeably. Similarly we use throughput and rate.

${ }^{2}$ Maximizing a weighted sum of the rates guarantees Pareto-optimality, while the specific selection of the weights enables controlling the trade-off between throughput and fairness [5].
} 


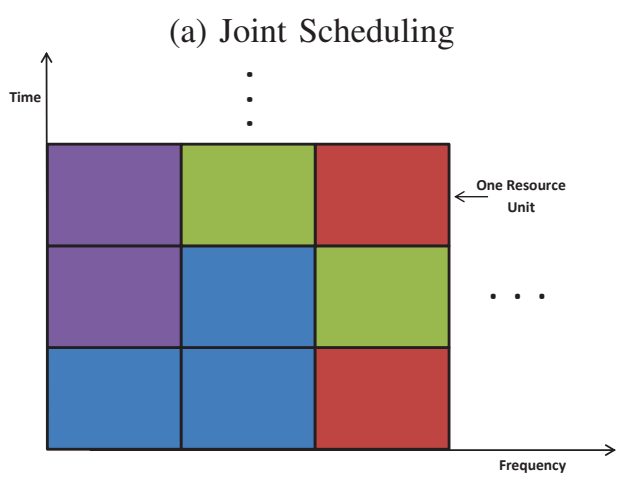

(b) User Scheduling Only

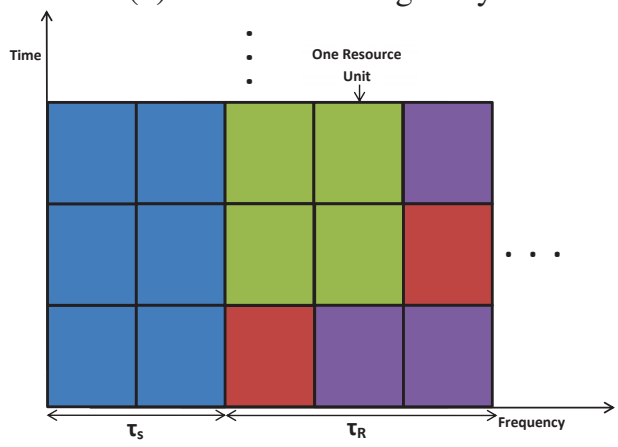

Fig. 2: Example of the allocation of RUs for (a) joint scheduling scheme (MUD+MHD gains) (b) User scheduling only (Buff+MUD gains). Blue represents the source (first hop), while red, green and purple represent three users.

is obtained because the relay does not have to immediately forward the data from the source to the intended user. Instead of that, it buffers the data and forwards it later when the user's channel is good. However, we do not obtain full MHD gains because a certain portion of the RUs are allocated to the S-R link regardless of the instantaneous channel values of the S-R link. The ratio of RUs where the source is scheduled is denoted by $\tau_{s}$, while the ratio of RUs in which the relay transmits to the users is denoted by $\tau_{R}$. The selection of $\tau_{s}$ and $\tau_{R}$ is based on maintaining the stability constraint. The RUs in $\tau_{R}$ are allocated opportunistically by selecting the $m$ th user to be scheduled according to (for constant power allocation)

$$
m=\arg \max _{i} \mu_{i} R_{i}[k] \quad i=1, \ldots, M,
$$

The average achievable rate by the source and $i$ th user can be written as $\bar{R}_{0}=\tau_{s} \bar{R}_{0}^{a c}$ and $\bar{R}_{i}=\tau_{R} \bar{R}_{i}^{a c}$ respectively, where

$$
\bar{R}_{0}^{a c}=\int_{0}^{\infty} r f_{R_{0}}(r) d r
$$

$\bar{R}_{i}^{a c}=\int_{0}^{\infty} r f_{R_{i}}(r) \operatorname{Prob}\left(i\right.$ th user is scheduled $\left.\mid R_{i}=r\right) d r$,

$$
\operatorname{Prob}\left(i \text { th user is scheduled } \mid R_{i}=r\right)=\prod_{l \neq i} F_{R_{l}}\left(\frac{\mu_{i} r}{\mu_{l}}\right) \text {, }
$$

where $f_{R}(r)^{3}$ is the probability density function (PDF) of the achievable rate and $F_{R}(r)$ is the cumulative distribution function (CDF) of it. $\tau_{s}$ and $\tau_{R}$ are adjusted such that the stability constraint is maintained. Therefore, we have $\tau_{s}+\tau_{R}=1$ and $\tau_{s} \bar{R}_{0}^{a c}=\sum_{i=1}^{M} \tau_{R} \bar{R}_{i}^{a c}$, which yields

$$
\tau_{s}=\frac{\sum_{i=1}^{M} \bar{R}_{i}^{a c}}{\sum_{i=1}^{M} \bar{R}_{i}^{a c}+\bar{R}_{0}^{a c}}, \quad \tau_{R}=\frac{\bar{R}_{0}^{a c}}{\sum_{i=1}^{M} \bar{R}_{i}^{a c}+\bar{R}_{0}^{a c}} .
$$

\section{B. Proportional Fair Scheduling}

To achieve proportional fairness (PF) [6], the users' weights are given by $\mu_{i}=\frac{1}{\bar{R}_{i}}$, which is in this case also equivalent to $\mu_{i}=\frac{1}{R_{i}^{a c}}$ since the ratios of the weights is the same in both cases. To obtain the optimal weights off-line, we substitute for $\bar{R}_{i}^{a c}$ using (5) and hence we get a total of $M$ equations and $M$ unknown $\mu_{i}$ 's. However, they are difficult to solve analytically. Therefore, we propose a recursive algorithm to obtain the optimal $\mu_{i}$ 's by simulating a real-time implementation of the PF scheduler. The update equation for $\mu_{i}$ is given by

$$
\mu_{i}[n]=\frac{1}{\bar{R}_{i}[n]+d[n]},
$$

where $n$ represents the iteration number (which is equivalent to the time index in real-time implementation), $d[n]=1-\frac{n}{1000}$ and $\bar{R}_{i}[n]$ is the average rate of user $i$ for all iterations up to $n$. The function $d[n]$ is used because average throughputs of all the users are initialized to zero. $d[n]$ is set to zero after the thousandth (selected arbitrary) iteration. The $\mu_{i}$ 's are updated in each iteration and these updated $\mu_{i}$ 's are then used to schedule the users in the next iteration and so on until we converge to the steady state value for the optimal weights for PF. The complete algorithm is shown in Algorithm 1.

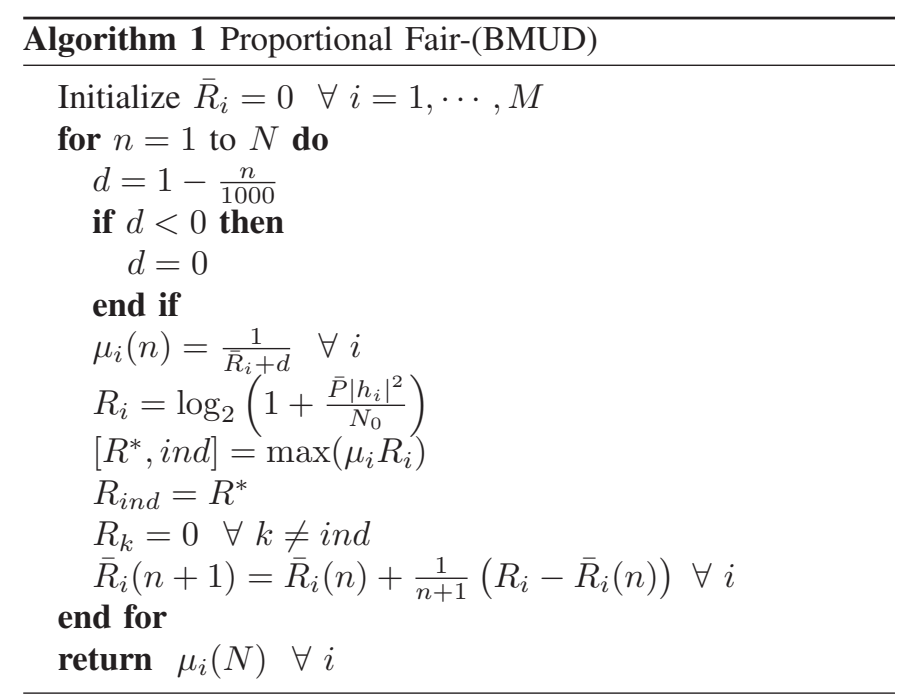

${ }^{3}$ For Rayleigh fading, $f_{R}(r)=\frac{\ln 2}{\rho} 2^{r} \exp \left(-\frac{2^{r}-1}{\rho}\right) \quad r \geq 0$, and $F_{R}(r)=1-\exp \left(-\frac{2^{r}-1}{\rho}\right) \quad r \geq 0$, where $\rho$ is the SNR [7]. 


\section{JOINT USER-AND-HoP SCHEDULING}

\section{A. Constant Power Allocation}

In this section, we show that the optimization problem in (2) can be maximized by joint scheduling the source and the $M$ user, hence exploiting multi-hop diversity. We can write the constrained problem in (2) as an constrained using the Lagrange dual problem as

$$
\frac{1}{K} \sum_{k=1}^{K} \max \sum_{i=1}^{M}\left(\mu_{i}-\mu_{0}\right) R_{i}[k]+\mu_{0} R_{0}[k],
$$

where we have assumed constant power per block (optimal power allocation is considered in the next subsection). We observe that the Lagrangian dual variable $\mu_{0}$ for the stability constraint appears as a weighting factor for the source. Furthermore, to maximize the weighted sum of the rates on the longterm, the optimal solution involves maximizing the weighted sum of the achievable rates for each RU independently. We can see from (9) that maximizing the initial problem in (2) is equivalent to, assuming half-duplex relaying and single user selection per block, selecting the source or the user with the maximum weighted instantaneous rate in each RU where the source weight, $\mu_{0}$, is optimized to maintain the stability constraint in (2). The selection criteria can be written as, where we use index 0 for the source,

$$
m=\arg \max _{j} \mu_{j}^{\prime} R_{j}[k] \quad j=0,1, \ldots, M
$$

where

$$
\mu_{j}^{\prime}= \begin{cases}\mu_{0} & j=0 \\ \left(\mu_{j}-\mu_{0}\right)^{+} & j=1,2 \ldots, M,\end{cases}
$$

where $(x)^{+}=\max (x, 0)$. Therefore, either the source or one of the users is scheduled in each RU depending upon (10) as shown in Fig. 2(a). So, unlike the scheme in Section III, there is no specific ratio of blocks in which the source is scheduled. It depends merely on the instantaneous channel conditions.

The long term achievable rates are given by

$$
\begin{gathered}
\bar{R}_{0}=\int_{0}^{\infty} r f_{R_{0}}(r) \operatorname{Prob}\left(\text { source is scheduled } \mid R_{0}=r\right) d r \\
\bar{R}_{i}=\int_{0}^{\infty} r f_{R_{i}}(r) \operatorname{Prob}\left(i \text { th user is scheduled } \mid R_{i}=r\right) d r
\end{gathered}
$$

From (10), we can obtain

$$
\operatorname{Prob}\left(\text { source is scheduled } \mid R_{0}=r\right)=\prod_{i=1}^{M} F_{R_{i}}\left(\frac{\mu_{0} r}{\mu_{i}^{\prime}}\right)
$$

$\operatorname{Prob}\left(i\right.$ th user is scheduled $\left.\mid R_{i}=r\right)=$

$$
F_{R_{0}}\left(\frac{\mu_{i}^{\prime} r}{\mu_{0}}\right) \prod_{l \neq i, 0} F_{R_{l}}\left(\frac{\mu_{i}^{\prime} r}{\mu_{l}^{\prime}}\right) .
$$

The optimal value of $\mu_{0}$ can be obtained by solving $\bar{R}_{0}=$ $\sum_{i=1}^{M} \bar{R}_{i}$, where $\bar{R}_{0}$ and $\bar{R}_{i}$ are obtained using (12) and (13) respectively. This problem can be solved numerically using a one-dimensional bisection search over $\mu_{0}$.

\section{B. Optimal Power Allocation}

Up till now, constant power per RU has been considered. We consider now optimal power allocation. Due to the additional constraint, the equivalent dual problem is given by

$$
\frac{1}{K} \sum_{k=1}^{K} \max \sum_{i=1}^{M}\left(\mu_{i}-\mu_{0}\right) R_{i}[k]+\mu_{0} R_{0}[k]-\sum_{j=0}^{M} \frac{\lambda P_{j}[k]}{N_{0}},
$$

where $\lambda$ is the dual variable which should be adjusted to maintain the average power constraint. Similar to the constant power case, the scheduling problem is equivalent to the conventional single-hop case by treating the source as a virtual user with weighting factor $\mu_{0}$. The scheduling rule in the $k$ th $\mathrm{RU}$ is now done according to

$$
m=\arg \max _{j}\left(\mu_{j}^{\prime} R_{j}[k]-\frac{\lambda P_{j}[k]}{N_{0}}\right) j=0,1, \ldots, M,
$$

where $\mu_{j}^{\prime}$ is defined in (11), and $P_{j}[k]$ is given by

$$
P_{j}[k]= \begin{cases}N_{0}\left[\frac{\mu_{j}^{\prime}}{\lambda}-\frac{1}{\left|h_{j}[k]\right|^{2}}\right]^{+} & j=m \\ 0 & \text { otherwise }\end{cases}
$$

To characterize the long-term rates, we use the PDF and CDF of the channel power gain, $|h|^{2}$, denoted respectively as $f_{h}(x)$ and $F_{h}(x)^{4}$ instead of $f_{R}(r)$ and $F_{R}(r)$ since the variable power allocation alters the statistics of the achievable rate. The single-user scheduling with optimal power allocation for conventional broadcast channel was studied in [8]. We apply the derived equations there to our case as follows. The power price parameter $\lambda$ can be obtained from the total average power constraint given by

$$
N_{0} \sum_{i} \int_{\frac{\lambda}{\mu_{i}^{\prime}}}^{\infty} f_{h_{i}}(x) \prod_{j \neq i} F_{h_{j}}(\zeta)\left[\frac{\mu_{i}^{\prime}}{\lambda}-\frac{1}{x}\right] d x=\bar{P},
$$

which can be solved numerically using a bisection search, where $\zeta$ equals

$$
\zeta=\frac{-\lambda}{\mu_{j}^{\prime} W\left[\left(-\frac{\lambda}{\mu_{i}^{\prime} x}\right)^{\frac{\mu_{i}^{\prime}}{\mu_{j}^{\prime}}} \exp \left(\frac{\mu_{i}^{\prime}}{\mu_{j}^{\prime}}-\frac{\lambda}{\mu_{j}^{\prime} x}-1,\right)\right]}
$$

and $W[$.$] is the Lambert function. The long term achievable$ rates are given by

$$
\begin{aligned}
& \bar{R}_{0}=\int_{0}^{\infty} \log _{2}\left(\frac{\mu_{0} x}{\lambda}\right) f_{h_{0}}(x) \prod_{i=1}^{M} F_{h_{i}}(\zeta) d x \\
& \bar{R}_{i}=\int_{0}^{\infty} \log _{2}\left(\frac{\mu_{i}^{\prime} x}{\lambda}\right) f_{h_{i}}(x) \prod_{j \neq i}^{M} F_{h_{j}}(\zeta) d x .
\end{aligned}
$$

${ }^{4}$ For Rayleigh fading, $f_{h}(x)=\frac{1}{\bar{\gamma}} \exp \left(-\frac{x}{\bar{\gamma}}\right), F_{h}(x)=1-\exp \left(-\frac{x}{\bar{\gamma}}\right)$, where $\bar{\gamma}$ is the average channel power gain. 
Similar to the constant power case, $\mu_{0}$ is obtained by equating the average source rate to the sum of the average users' rates.

\section{Real Time Adaptation of Weighting Factor $\mu_{0}$}

In the discussion up till now, it has been assumed that the PDF and CDF of the S-R and all R-D channels are perfectly known, and hence off-line calculation of $\mu_{0}$ is done. However, this might not be always feasible in practice. Therefore, we propose an alternative approach to obtain the source weighting factor, $\mu_{0}$, based on real time channel measurements similar to the one adopted in [9] in the context of maintaining fairness constraints. The scheduling criteria remains the same as in (10). $\mu_{0}$ is updated with each time index $n$ according to

$\mu_{0}(n+1)=\mu_{0}(n)+\frac{\delta}{n+1}\left(\sum_{i=1}^{M} \bar{R}_{i}(n+1)-\bar{R}_{0}(n+1)\right)$,

where $\delta$ controls the convergence speed of the algorithm. In our numerical results, we used $\delta=2$. The complete algorithm is given in algorithm 2 .

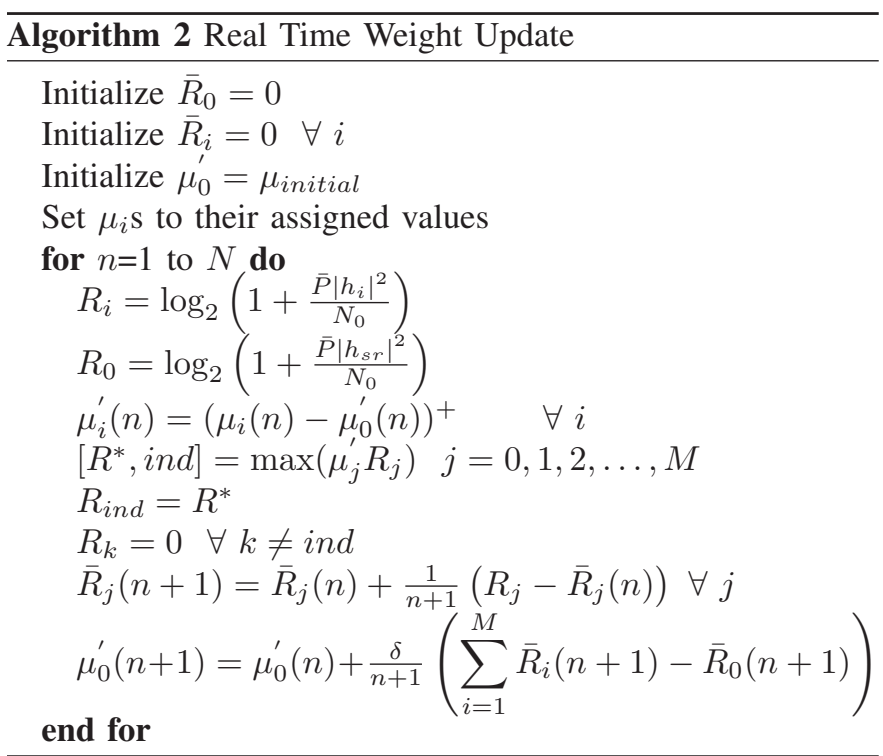

\section{Proportional Fair Scheduling}

We consider PF scheduling scheme for joint scheduling as we did for multi-user scheduling. The recursive algorithm is similar to Algorithm 1. However, the selection criteria changes and the source becomes considered with the users for scheduling over all RUs. The scheduling criteria and the update of $\mu_{j}^{\prime}$ are the same as Algortihm 2. The update equation for $\mu_{0}$ is given by (23).

\section{Numerical Results}

We present numerical results to the different schemes discussed in this paper. We compare joint scheduling (in Section IV), which offers full MHD and MUD gains, with the users-only scheduling scheme (in Section III), which offers buffering and MUD gains but not full MHD gains.

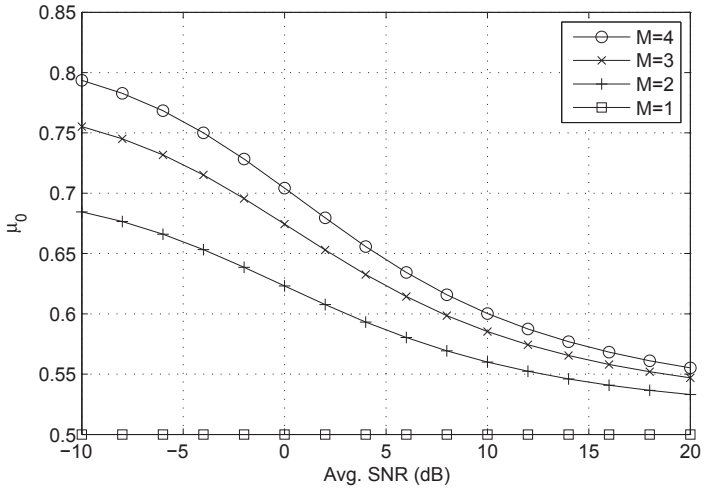

Fig. 3: Optimal $\mu_{0}$.

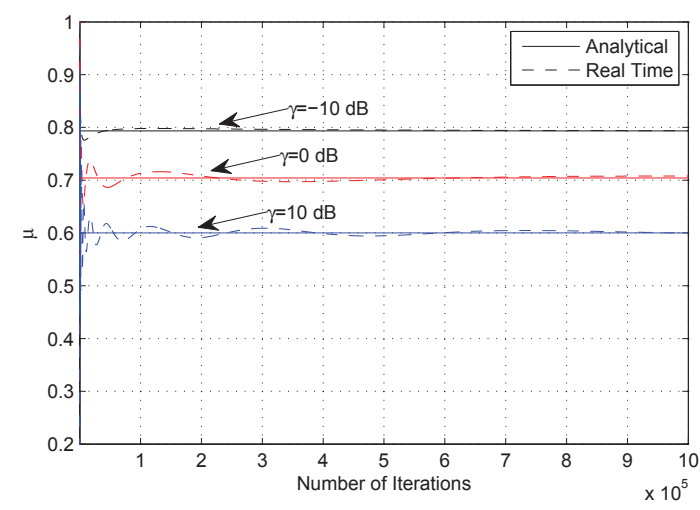

Fig. 4: Real time update of optimal $\mu_{0}$ for $M=4$.

We commonly use the long term source throughput in the comparisons, which does also refer to the sum throughput of all users. We use Rayleigh block-fading channel model for the S-R and all R-D channels.

We start by considering the case of independent and identically distributed (IID) channels for all links in the first and second hops. We consider the case of maximum sum throughput scheduler, and hence we set $\mu_{i}=1$ for all users. The optimal value of $\mu_{0}$ as a function of average signalto-noise-ratio (SNR) of all channels is shown in Fig. 3 for different number of users. The optimal value of $\mu_{0}$ decreases with increasing Avg. SNR because at low SNR the source is scheduled more frequently. Fig. 4 shows the real-time approach to obtain $\mu_{0}$ and compares it with the analytical value obtained by offline calculation. We can see from Fig. 4 that the real time algorithm converges to the analytical solution. $\gamma$ in Fig. 4 is defined as $\gamma=\frac{\bar{P}}{N_{0}}$.

The long term source (sum of all users) throughput, $\bar{R}_{0}$, for the different schemes are plotted in Fig. 5 for two, eight and sixteen users with IID channels. It is clear from Fig. 5 that joint scheduling gives the best performance as we would expect due to the MHD gains. Additionally, it is evident from Fig. 5 that the gain in performance due to increase in number of users deceases at large number of users. Fig. 6 shows a comparison between constant power (CP) and optimal power allocation (OPA) per RU for $M=1$ and $M=4$ with IID channels. We observe that OPA provides gains at low Avg. SNR only while the gains are negligible for medium or high 


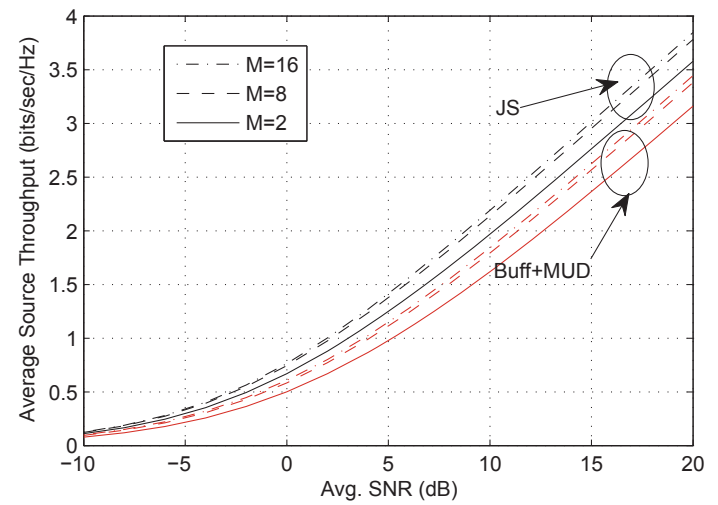

Fig. 5: Comparison of average source throughput, $\bar{R}_{0}$, with $M=2,8$ and 16 .

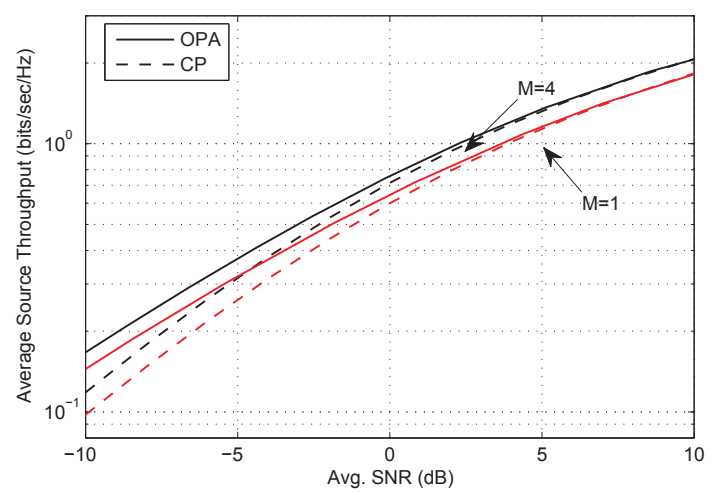

Fig. 6: Comparison between OPA and CP for joint scheduling.

SNR. Therefore, applying constant power allocation is in most cases sufficient and it is also simpler to implement.

The gains of joint scheduling over users-only scheduling are also evident in the case of PF scheduling as shown in Fig. 7 which is for a two user case with different average channel values. The advantages of JS are also confirmed by characterizing the two-user rate region which is shown in Fig. 8. The rate region is obtained by scanning all possible user weights from $\left(\mu_{1}=1, \mu_{2}=0\right)$ to $\left(\mu_{1}=0, \mu_{2}=1\right)$. Joint scheduling enlarges the rate region due to the MHD gains.

\section{Vi. CONClusions}

We have proposed and investigated joint scheduling for dual-hop block-fading broadcast channels to maximize the weighted sum of the long term user throughputs. We have compared joint scheduling to a conventional scheduler with multi-user scheduling only and we have demonstrated via numerical examples that joint scheduling provides performance improvement in terms of long term achievable rate region. We have also proposed recursive algorithms to obtain the source and user weights for proportional fair scheduling for both cases of joint scheduling and multi-user scheduling. The gains of joint user-and-hop scheduling over user-only scheduling are obtained because it exploits multi-hop diversity in addition to multi-user diversity. Due to these gains, we believe that our proposed scheduling scheme is favorable for practical implementation in next generation wireless systems that deploy relays such as in LTE-Advanced systems.

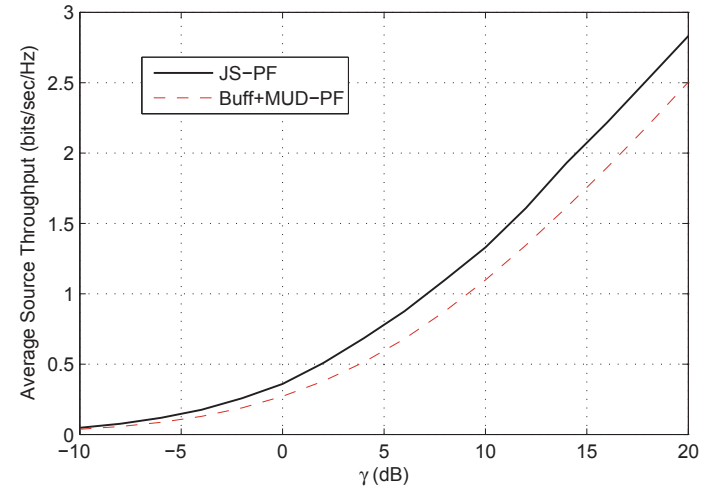

Fig. 7: Comparison of average source throughput for proportional fair scheduling, with $\bar{\gamma}_{0}=0.4, \bar{\gamma}_{1}=1$ and $\bar{\gamma}_{2}=0.1$.

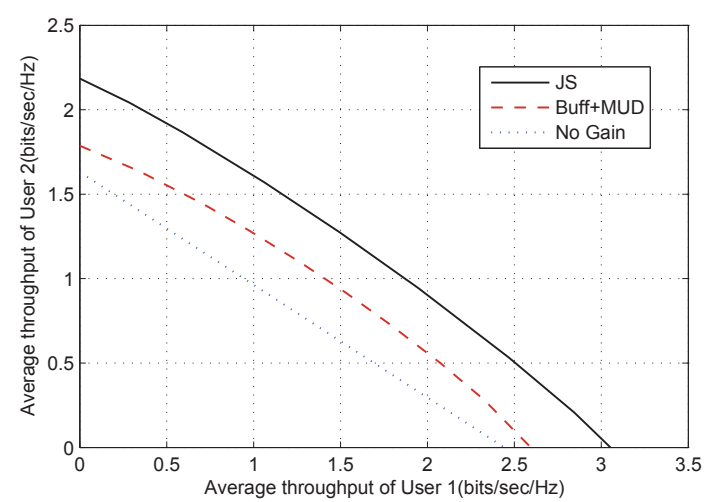

Fig. 8: Comparison of capacity regions for $\gamma=20 \mathrm{~dB}$, with $\bar{\gamma}_{0}=$ $0.4, \bar{\gamma}_{1}=1$ and $\bar{\gamma}_{2}=0.1$.

\section{REFERENCES}

[1] Y. Yang, H. Hu, J. Xu, and G. Mao, "Relay technologies for WiMAX and LTE-Advanced mobile systems," IEEE Communications Magazine, vol. 47, no. 10, pp. 100-105, Oct. 2009.

[2] H. Ekstrom, "QoS control in the 3GPP evolved packet system," IEEE Communications Magazine, vol. 47, no. 2, pp. 76-83, Feb. 2009.

[3] M. Shaqfeh and H. Alnuweiri, "Joint power and resource allocation for block-fading relay-assisted broadcast channels," IEEE Transactions on Wireless Communications, vol. 10, no. 6, pp. 1904-1913, Jun. 2011.

[4] W. Mesbah and T. Davidson, "Power and resource allocation for orthogonal multiple access relay systems," EURASIP Journal on Advances in Signal Processing, vol. 2008, pp. 1-15, article ID 476125.

[5] M. Shaqfeh and N. Goertz, "Performance analysis of scheduling policies for delay-tolerant applications in centralized wireless networks," in Proceedings IEEE International Symposium on Performance Evaluation of Computer and Telecommunication Systems (SPECTS'08), Edinburgh, UK, Jun. 2008, pp. 309-316.

[6] P. Viswanath, D. Tse, and R. Laroia, "Opportunistic beamforming using dumb antennas," IEEE Transactions on Information Theory, vol. 48, no. 6 , pp. 1277-1294, Jun. 2002.

[7] B. Hogstad, M. Patzold, N. Youssef, and V. Kontorovitch, "Exact closedform expressions for the distribution, the level-crossing rate, and the average duration of fades of the capacity of OSTBC-MIMO channels," IEEE Transactions on Vehicular Technology, vol. 58, no. 2, pp. 10111016, Feb. 2009.

[8] M. Shaqfeh, N. Goertz, and J. Thompson, "Ergodic capacity of blockfading Gaussian broadcast and multi-access channels for single-userselection and constant-power," in Proceedings $17^{\text {th }}$ European Signal Processing Conference (EUSIPCO'09), Glasgow, Scotland, UK, Aug. 2009, pp. 784-788.

[9] M. Shaqfeh and N. Goertz, "Channel-aware scheduling with resource -sharing constraints in wireless networks," in Proceedings IEEE International Conference on Communications (ICC'2008), Beijing, China, Jun. 2008, pp. 4149-4153. 